\title{
Formal Security Analysis and Improvement of a hash-based NFC M-coupon Protocol
}

\author{
No Author Given \\ No Institute Given
}

\begin{abstract}
Near field communication (NFC) is a Radio Frequency (RF) technology that allows data to be exchanged between devices that are in close proximity. We formally analyse a hash based NFC mobile coupon protocol using formal methods (Casper/FDR2). We discover a few possible attacks which break the requirements of the protocol. We propose solutions to address these attacks based on two different threat models. In addition, this paper is a facilitating case study for a black-box user to become a more powerful analyser.
\end{abstract}

Keywords: NFC, M-coupon, CasperFDR, Formal verification, Protocol security

\section{Introduction}

Near Field Communication (NFC) [1] is a radio frequency (RF) communication link, which allows data to be exchanged between devices that are normally less than $10 \mathrm{~cm}$ apart [2]. NFC-based mobiles are an emerging technology changing the way we communicate with objects. For instance, payments, tickets and coupons can be exchanged just by waving the NFC-based mobile at the points of sale.

NFC security is an important issue that has been emphasised in the literature $[3,4]$. Even though NFC has the advantage of a short communication link, security measures must be considered especially with sensitive applications to address security requirements, such as confidentiality, integrity and availability.

The NFC mobile coupon application (M-coupon) is one of the promising and popular applications [5-8]. An M-coupon is a cryptographically secured electronic message with some value. It requires secure issuing and cashing of the M-coupons, otherwise it can cause huge losses for a company and damage its reputation [9].

The NFC M-coupon system has a typical scenario, see Figure 1. All parties have NFC capability, in order to communicate with each other. Firstly, a user scans his NFC mobile against an NFC issuer (e.g., a smart poster or newspaper). Then, an M-coupon is issued and sent to his mobile. Later, the user goes to the shop to cash the M-coupon with the cashier. The cashier may authenticate the user before the cashier provides the promised bonus. Only the cashier needs to have online access, whereas the issuer and the user can both be offline. Hsiang 


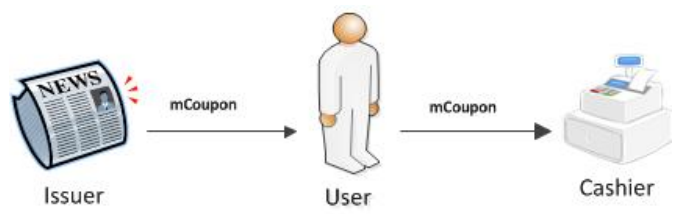

Fig. 1. General NFC mobile coupon

et al. [10] have proposed a secure hash-based M-coupon protocol which allows secure issuing and cashing of M-coupons. They designed the protocol to address specific M-coupon requirements.

On the other hand, designing a security protocol is a difficult task even with strong encryption methods. Many attacks may be possible on the cryptographic protocols just by intercepting and replaying encrypted messages between entities, without decrypting any messages. Formal security analysis is a powerful approach to check the security of a protocol and whether it address its requirements [11].

In this paper we use the CasperFDR approach [12] based on Communicating Sequential Processes (CSP) [13], a formal method (state exploration) approach, to formally analyse the NFC M-coupon protocol proposed by Hsiang et al [10]. Our analysis found attacks against the protocol. We then provide three solutions to address these vulnerabilities. We formally verify with CasperFDR that the flaw is addressed. In fact, this paper also provides a case study for a black-box user who wish to become more powerful in protocol analysis by CasperFDR.

\section{The Casper Approach}

In our analysis we use CSP [13], with its model checker Failures Divergence Refinement (FDR2), which is proven to be an effective method in analysing the security of protocols [14]. However, modelling protocols in CSP is not a trivial task. Gavin Lowe developed CasperFDR [12], a tool that allows the user to write an abstract description of a security protocol, then the tool produces codes in CSP language, and directly checks it with FDR2. CasperFDR has been used to analyse a huge number of protocols [15], which proves its capability of finding vulnerabilities, making it a robust CSP model producer.

CasperFDR is a formal method tool which supports symbolic protocol analysis in the Dolev-Yao model [16] which assumes no encrypted message can be decrypted without the decryption key, thus the CasperFDR intruder model does not perform any cryptanalysis. However, the intruder does have full control of the network traffic, and tries to break the security protocol from what has been learned.

The way attacks are found by a refinement checking of protocol's properties. When refinement fails, then it provides a trace which show how the property fails, that correspond to an attack. 


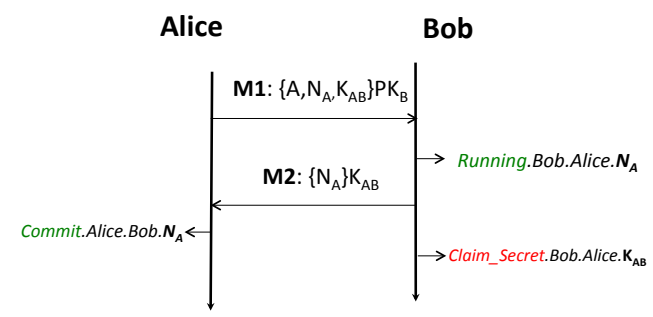

(a) Simple protocol

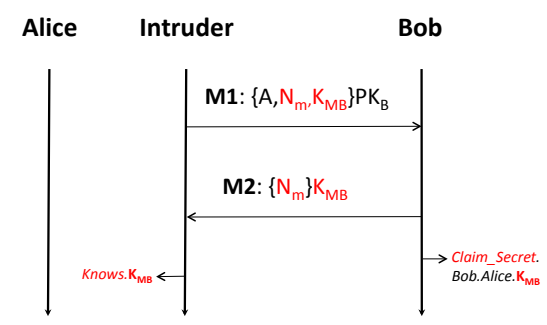

(b) Simple protocol attack

Fig. 2. Illustrating example

\subsection{Simple example}

Figure $2 \mathrm{a}$ is our demonstrating simple protocol. A two message protocol aims to authenticate Bob to Alice:

1. Alice $\rightarrow$ Bob $:\left\{A, N_{A}, K_{A B}\right\} P_{B}$

2. Bob $\rightarrow$ Alice $:\left\{\mathrm{N}_{\mathrm{A}}\right\} \mathrm{K}_{\mathrm{AB}}$

Message 1 sent by Alice to Bob containing Alice identity, Nonce (number used once) and a session key $\mathrm{K}_{\mathrm{AB}}$, encrypted with Bob's public key. Then, Bob send message 2 by encrypting the Nonce $\left(\mathrm{N}_{\mathrm{A}}\right)$ with the session key. Alice authenticates Bob based on the fact that since message 1 is encrypted by Bob's public key, he is the only one can extract the session key and the nonce and send message 2. At the end of the protocol both Alice and Bob believe the session key $\mathrm{K}_{\mathrm{AB}}$ is secret . 
Analysing this protocol in CasperFDR is a quite straightforward procedure. Having modelled the exchanged messages between entities, we check the claimed authentication and secrecy using the following claims:

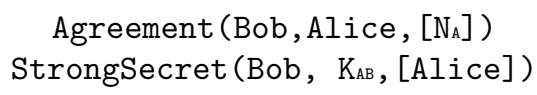

The Agreement specification means it will check whether Bob is authenticated to Alice and have both of them agreed on the Nonce $\mathrm{N}_{\mathrm{A}}$. The StrongSecret specification is checking whether the key $\mathrm{K}_{\mathrm{AB}}$ is secret between Bob and Alice. CasperFDR will complete the remaining process for us as we explained earlier.

Nevertheless, understanding how these specifications are captured underneath CasperFDR is important if we want precise descriptions of how claimed properties are modelled in a specific application, as we will see later.

Capturing authentication between Alice and Bob in the protocol is done by utilising new events injected in the protocol as demonstrated in Figure 2a. These events are Running and Commit. Initially, Alice and Bob are modelled as independent CSP processes. After message 1, Bob performs the Running event, which means Bob starts running the protocol apparently with Alice. Then, Alice will perform the Commit event at the end of her part of the protocol, which means Alice has finished a run of the protocol with Bob. Alice could make sure she was running the protocol with Bob based on the fact that if Alice reaches the Commit event then Bob must have reached the Running event before. Launching an attack relies on the possibility of the intruder, without taking Bob's role, to engineer a trace of the protocol in which Alice runs the Commit event without a corresponding Running event from Bob.

For secrecy, only the Claim_Secret event is used by Alice and Bob. Figure 2a only shows when Bob performs Claim_Secret event. An attack is launched if the intruder could break this claim, by finding a trace of the protocol in which the intruder knows a claimed secret, without taking Alice's or Bob's roles.

The Running, Commit and Claim_Secret events can also contain more information specific to the agreement required between the participants. They are constructed by:

$$
\text { Agent.Agent.Message }
$$

For example Running.Bob.Alice. $N_{A}$, which means Bob starts a run of the protocol, apparently with Alice, using nonce $\mathrm{N}_{\mathrm{A}}$.

The Casper analysis finds no attack on authentication, but there is an attack on secrecy. Figure $2 \mathrm{~b}$ illustrates how an intruder can creak a session key that Bob believes is secret with Alice. Anyone can generate message 1 since Bob's public key is publicly known. The intruder impersonates Alice by including Alice identity, making Bob believe the session key $\mathrm{K}_{\mathrm{MB}}$ is secret with Alice.

\subsection{Hierarchy of Authentication and secrecy}

CasperFDR provides different flavours of testing authentication and secrecy. The strongest form of authentication specification is Agreement. If Alice and 
Bob meet the Agreement specification, then if Bob thinks he has successfully completed a run of the protocol with Alice, then Alice has previously been running the protocol, agreeing on their roles in the protocol, and there is a oneto-one relationship between Alice and Bob i.e. each run of Alice corresponds to a unique run of Bob.

A weaker authentication specification is NonInjectiveAgreement. The difference from Agreement is that the one-to-one relationship is not required. Each run of one participant matches a run of the other but they can overlap. For example, two "Commit" events may correspond to the same "Running" event.

Secrecy has two forms of specification, Secret and StrongSecret. Secret is testing if the intruder could know the secret value at the end of the protocol. StrongSecret is stronger than Secret that it even checks whether the intruder is able to know the secret value without completing a full run of the protocol.

\subsection{Channels}

CasperFDR allows adjusting the intruder's ability on any messages of the protocol. For example:

\#Channels

1 NF NRA- NR

2 C NF NRA NR

The first line means that on message 1 the intruder neither can fake data $N F$ , nor honest reascribing $N R A$ - (changing the sender ID except to his own ID) nor redirecting $N R$ (changing the receiver ID). The second line means that on message 2 the intruder neither can eavesdrop $C$, nor fake data, nor reascribing nor redirecting.

By adjusting some of protocol's channels, we can capture assumptions made in the protocol as we will see later.

\section{Protocol Security Requirements}

The analysed protocol we consider in this paper intends to address six security requirements, as stated by the protocol designer in [10]:

- Confidentiality: A third party shall not be able to obtain the M-coupon by eavesdropping.

- Data Integrity: An attacker shall not be able to modify data during the communication.

- Forgery Protection:

- No Unauthorized Generation: An attacker shall not be able to issue his own M-coupon.

- No Manipulation: M-coupon shall not stay valid after a manipulation.

- Unauthorized Copying: An attacker shall not be able to produce a valid copy of an M-coupon and cash it in. This requirement can be divided into: 
- Not Transferable: Whatever identity is presented at issuing phase shall not be changed during the protocol.

- User Authentication: in addition to Not transferable, the identity of the user is the one who it claim to be. The user who issued the M-coupon must be the one who is cashing it at the cashier. This requires the cashier to authenticates the user through some authentication methods.

This protocol only addresses the Not transferable requirement.

- No Multiple Cash-in: An attacker shall not be able to use the same Mcoupon multiple times.

\subsection{Formal definition}

Figure 3 illustrates a formal definition of these requirements which are required for series of protocols, and the relationship between them.

Confidentiality requires that data representing the M-coupon in the protocol must satisfy StrongSecret specification between the issuer and the cashier.

NonInjectiveAgreement specification includes three requirements, and layers, of authentications between the cashier and the issuer. We are not concern here with repeats because it is checked by other requirement and it may be not required in some systems. Forgery protection, after identifying data representing the M-coupon, a NonInjectiveAgreement on the M-coupon between the issuer and the cashier is required. This is violated if the cashier accepts the M-coupon that have not been issued by the issuer. This implies either that the M-coupon have been created by an attacker (i.e. Unauthorised Generation) or else that the M-coupon generated by the issuer has been modified to another (i.e. No Manipulation ). Not Transferable is a stronger specification than forgery protection that it also requires an agreement on a user identity attached to the M-coupon. The strongest NonInjectiveAgreement specification is Data Integrity, both the cashier and the issuer must agree on the integrity of all data in the protocol.

No Multiple Cash-in requires an Agreement specification between the issuer and the cashier. Every time the cashier accepts M-coupon, there must be a separate occasion where the issuer must have issued them. Hence cashier cannot accept M-coupon more times than issuer sent them.

User Authentication is an Agreement between the user and the cashier on some credential. Even though the M-coupon might be used many times, user must be authenticated in each time.

\section{Protocol Description}

The M-coupon protocol of [10] uses simple hash functions, which is a computationally light cryptographic method, a suitable choice with standard RFID/NFC tags. The notation in the protocol description is as follows: 


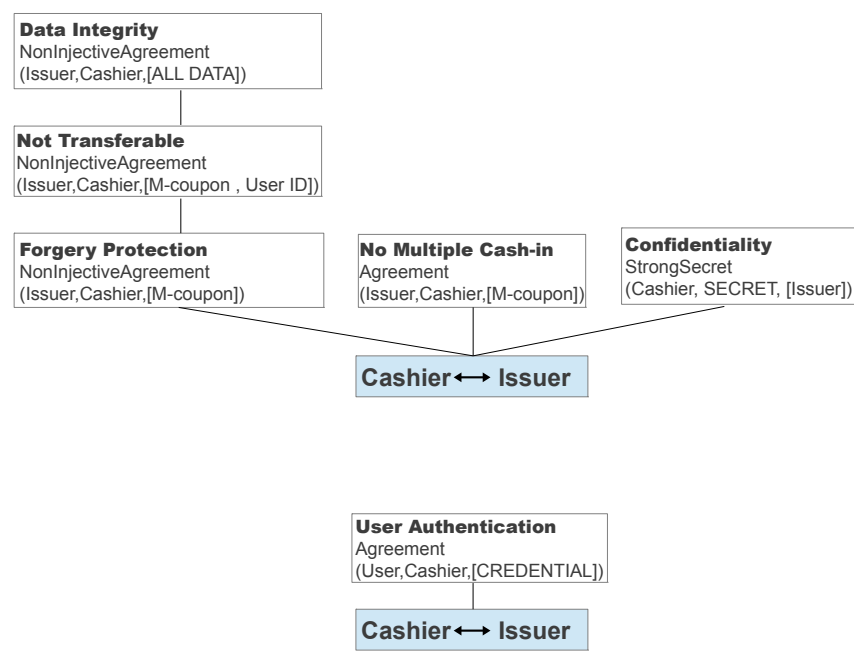

Fig. 3. Hierarchy of authentication/secrecy properties

ID(i) Issuer ID

$I D(u)$ User ID

Offer The promised Offer with required data

$X \quad$ A secret key between the issuer and the cashier

$\oplus \quad$ Exclusive or (XOR)

$h[.$.$] \quad Hash function$

There are four messages in this protocol:

1. $U \rightarrow I: I D(u)$

2. $I \rightarrow U: M=\{I D(u), V, C\}$

3. $U \rightarrow C: M=\{I D(u), V, C\}$

4. $C \rightarrow U: B O N U S$

Initially, the cashier $C$ and the issuer $I$ share a secret value, $X$ and an offer. The cashier stores a table consisting of hashes of all issuers identities, $h[I D(i)]$.

At the issuing phase, the user's mobile $U$ sends his identity to the issuer (message 1). Then, the issuer produces a coupon, $M$ (message 2), consisting of three parts: $I D(u), V$ and $C$.

$$
\begin{aligned}
& V=I D(u) \oplus h[I D(i)] \\
& C=h[h[I D(i)] \oplus X \oplus \text { Offer }]
\end{aligned}
$$

At the cashing phase, the user's mobile sends the M-coupon to the cashier (message 3). Then, the cashier obtains $h[I D(i)]$ by computing $I D(u) \oplus V$. The cashier can look up ID(i) from the hash, and then find $X$ and Offer. When 
the cashier has $I D(u)$ and Offer, it can decide if the M-coupon has been used before, and reject it accordingly. Then, the cashier will check the validity of the M-coupon by computing $h[h[I D(i)] \oplus X \oplus$ Offer $]$ and confirm it matches $C$. The cashier stores $I D(u)$ to prevent re-use of the coupon, and sends the Bonus to the user.

The intention is that the Confidentiality and Data Integrity requirements are ensured by use of the secret value $X$. By including the identity of the user's mobile and offer, Multiple Cash-in and Not Transferable can be managed. Forgery Protection is addressed by the secret value $X$ which is known only by the cashier and the issuer.

\section{$5 \quad$ Modelling}

In order to capture the NFC M-coupon requirements in the protocol, we develop two main models shown in Figure 4. The main model Figure 4a captures four requirements: Confidentiality, Forgery Protection, Not Transferable and Data Integrity. The second model illustrated in Figure 4b captures the No Multiple Cash-in requirement. Moreover, data representing the M-coupon in the protocol are $X$ and Offer.

\subsection{The protocol's requirements}

We show in the following sections what we have to write in CasperFDR to model the protocol's requirements of section 3, then how it is captured underneath in terms of Running, Commit and Claim_Secret events. This enabling us to have formal and precise descriptions of capturing NFC mobile coupon requirements.

Confidentiality We model confidentiality in Casper as follows:

$$
\begin{gathered}
\text { StrongSecret (C, X , [I]) } \\
\text { StrongSecret (C, Offer , [I]) }
\end{gathered}
$$

These secrecy specifications means the cashier $C$ claims that $X$ and $O f f e r$ are confidential between the cashier $C$ and the issuer I. StrongSecret checks whether the intruder is able to break these claims without completing the protocol.

Figure 4a illustrates the first specification, secrecy of $\mathrm{X}$. When the cashier $C$ performs the Claim_Secret.C.I.X event, it can expect $X$ to be a secret with the issuer $I$ who shares the secret key $X$. If this is violated then the intruder can complete a run of the protocol with the cashier without taking the issuer role in the protocol, and learn the secret key $X$. A similar description for the Offer specification.

Forgery Protection We model forgery protection in Casper as follows:

$$
\text { NonInjectiveAgreement (I, C, [X,Offer]) }
$$


We identify the M-coupon with $X$ and Offer. This states that If cashier accepts $X$ and Offer, then the issuer must have issued them. NonInjective means that it is not concerned with repeats. I.e. the cashier can accept many times what was issued once. This is violated if the cashier accepts $X$ and Offer that have not been issued by the issuer. This implies either that $X$ and Offer have been created by an attacker (i.e. Unauthorised Generation) or else that an M-coupon generated by the issuer has been modified to another (i.e. No Manipulation ). Hence if this property holds then we have Forgery Protection: No Unauthorised Generation and No Manipulation.

This is illustrated in Figure 4a. After the issuer completes its part of the protocol, it performs the Running.I.C.X.Offer event, which means the issuer $I$ starts a running of the protocol, apparently, with the cashier $C$, agreeing on $X$ and offer. Later, the cashier will perform the Commit.C.I.X.Offer event at the end of its part of the protocol, which means the cashier $C$ has finished the protocol with the issuer $I$, agreeing on the $X$ and Offer.

Unauthorized Copying (Not Transferable) We model Not Transferable in Casper as follows:

NonInjectiveAgreement (I, C, [X,Offer, ID (u)] )

This specification is similar to forgery protection specification, but also with an agreement on a user identity. The coupon, $[X, O f f e r]$, must be attached to one user only $I D(u)$. Both the issuer and the cashier agree on the user to use the coupon as many times as he like, as long as the coupon has been issued by a genuine issuer, and is being used by the intended user. An example for such coupon is frequent flyer coupon.

This is shown in Figure 4a. After the issuer completes its part of the protocol, it performs the Running.I.C.X.Offer.ID $(u)$ event, which means the issuer $I$ starts a running of the protocol, apparently, with the cashier $C$, agreeing on $X, O f f e r$ and $I D(u)$. Later, the cashier will perform the Commit.C.I.X.Offer.ID $(u)$ event at the end of its part of the protocol, which means the cashier $C$ has finished the protocol with the issuer $I$, agreeing on the $X$, Offer and $I D(u)$.

Observe that this property is stronger than forgery protection. If it holds then not only the m-coupon must be genuine, as for forgery protection, but it must also have the same user.

Data Integrity We model Data Integrity in Casper as follows:

$$
\text { NonInjectiveAgreement (I, C, [X, Offer, ID (u), ID (i)]) }
$$

This will check the integrity of the protocol. Both the cashier and the issuer must agree on all the information in the protocol. 


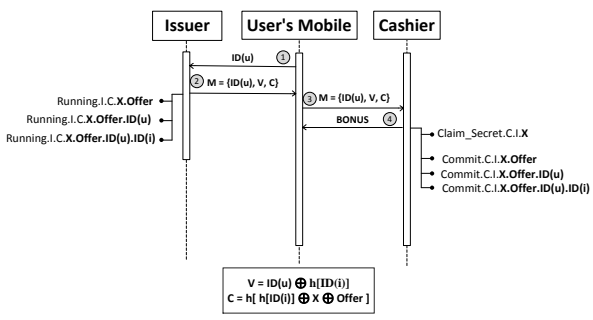

(a) Main model: Confidentiality, Forgery Protection, Not Transferable and Data Integrity

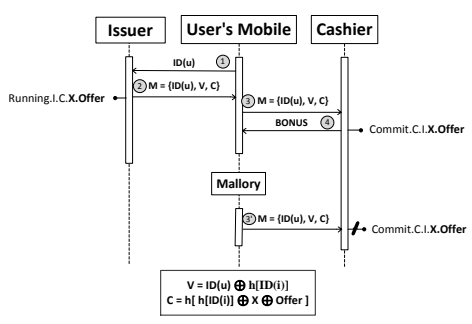

(b) No Multiple Cash-in model

Fig. 4. Capturing NFC M-coupon requirements

No Multiple Cash-in We model No Multiple Cash-in in Casper:

$$
\text { Agreement (I, C, }[\mathrm{X}, \text { Offer]) }
$$

This specification states that every time the cashier accepts $X$ and Offer, there must be a separate occasion where the issuer must have issued them. Hence cashier cannot accept $X$ and Offer more times than issuer sent them.

Figure 4b illustrates a scenario where the cashier is engaging in the protocol twice, with one issuer run. The first time the cashier runs the protocol with the user's mobile, and the second, illegal, time with Mallory who might be an intruder or the user himself. The second Commit should not occur if there was not a separate Running.

\subsection{Intruder knowledge}

The analysis also requires us to define the initial knowledge of the intruder. The intruder knows the following: the identities of himself, the user and the cashier, and the hash function.

\subsection{Assumptions}

There is an assumptions made by the protocol's designers that the client's ID is bounded to the client's mobile device, and therefore the client is authenticated at issuing and cashing phases. Therefore, we analyse this protocol in two different assumptions: as no assumption made (The Dolev-Yao model) and as the user's ID is bounded to the mobile.

The main goal for analysing the protocol under this assumption is that if an attack is discovered under the Dolev-Yao model, then we should examine if the attack still apply under the assumption made. 
We blind message 1 from the intruder i.e. the intruder can not eavesdrop, fake, re-ascribing or redirect message 1. We model this in Casper as follows:

\#Channels

1 C NF NRA NR

\section{Analysis}

The outcome of the analysis shows no attack in Confidentiality and Forgery Protection.

However, attacks were found in Not Transferable, Data Integrity and No Multiple Cash-in properties. The main vulnerability is a simple logical attack against the hashes of the M-coupon. The identity of the user attached to the M-coupon can be easily extracted and changed to any identity. If we consider the M-coupon, the identity $I D(u)$ is not attached correctly to the M-coupon. Anyone is able to compute the first two parts, $I D(u)$ and $V$ to get $h[I D(i)]$ :

$$
h[I D(i)]=I D(u) \oplus V
$$

By obtaining $h[I D(i)]$, the intruder is able to attach any identity, such as $I D$ (intruder) without changing the third part $C$, and thus produce a new coupon $M^{\prime}$ :

$$
\begin{aligned}
V^{\prime} & =I D(\text { intruder }) \oplus h[I D(i)] \\
M^{\prime} & =I D(\text { intruder }), V^{\prime}, C
\end{aligned}
$$

Even though this analysis was under the Dolev-Yao threat model, the properties are still broken under the assumption of a bounded user ID. The attacker still could change the user identity in an eavesdropped M-coupon to his own identity, and cash it in with the cashier. The intruder could even know the user ID by pretending to be an issuer.

As far as the analysis is concerned, the Unauthorized Copying property can be divided into two properties: Not Transferable and User Authentication. Not Transferable is an agreement between the issuer and the cashier that whatever user identity presented at issuing phase, it shall not be changed during the protocol. Whereas, User authentication is stronger in that, in addition to Not transferable, the identity of the user is the one who it claim to be. I.e. an agreement between the user and the cashier. This protocol only tries to address Not transferable requirement, which may be sufficient in the case of their assumption or in a secure and trusted issuing phase.

\section{Suggested solution}

We suggest three solutions to address the found vulnerability: An enhanced hashbased protocol which is a solution based on the assumption of the bounded ID 
Table 1. Hash based protocol and provided solutions against intended/addressed/failed requirements

\begin{tabular}{|l|c|c|c|c|}
\hline & Hash-based & Enhanced Hash-based & Footfall & Premium \\
\hline Confidentiality & $\sqrt{ }$ & $\sqrt{ }$ & $\sqrt{ }$ & $\sqrt{ }$ \\
\hline Forgery Protection & $\sqrt{ }$ & $\sqrt{ }$ & $\sqrt{ }$ & $\sqrt{ }$ \\
\hline Data Integrity & $\mathrm{x}$ & $\sqrt{ }$ & $\sqrt{ }$ & $\sqrt{ }$ \\
\hline No Multiple Cash in & $\mathrm{x}$ & $\sqrt{ }$ & $\sqrt{ }$ & $\sqrt{ }$ \\
\hline Not Transferable & $\mathrm{x}$ & $\sqrt{ }$ & & $\sqrt{ }$ \\
\hline User Authentication & & & & $\sqrt{ }$ \\
\hline
\end{tabular}

assumption. In addition, We provide two kinds of marketing-oriented M-coupon protocols, the footfall M-coupon protocol and the premium $M$-coupon protocol, both of which are based on the Dolev-Yao model. Table 1 summarises solutions provided with addressed properties.

\subsection{Enhanced hash-based protocol}

In order to address the broken properties ( Not Transferable, Data Integrity and No Multiple Cash-in) in the original hash-based protocol, the identity of the user must be attached correctly to the coupon. This solution must be only considered in a secure and trusted issuing phase. The change needed is replacing $C$ part in Figure ?? to become:

$$
C=h[h[I D(i)], X, O f f e r, I D(u)]
$$

As far as the Not Transferable property is concerned, it is only useful within a trusted issuing phase which is not always the case. The fact that user ID can be faked by anyone makes combining the User Authentication property with Not Transferable property more useful and meaningful. So, the best choice would be to use them all, the premium protocol, or drop them all, the footfall protocol.

\section{2 marketing-oriented protocols solutions}

The footfall M-coupon protocol is used when the main purpose of the M-coupon is to increase the number of people visiting the shop, regardless of whom is using it. Whereas, the premium M-coupon protocol used when the client has paid for it, and only the intended user is allowed to cash it. The premium Mcoupon protocol addresses all requirements discussed in section 3. The footfall M-coupon protocol addresses the same requirements, except Not Transferable and User Authentication.

The footfall M-coupon protocol Figure 5a shows the footfall M-coupon protocol. 


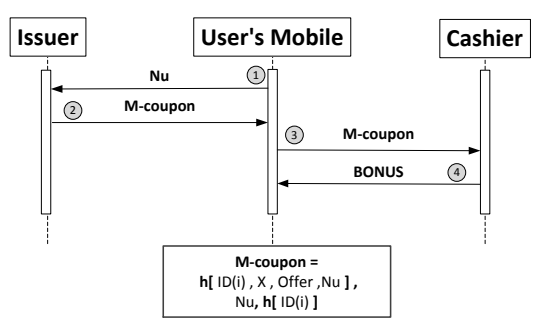

(a) the footfall M-coupon protocol

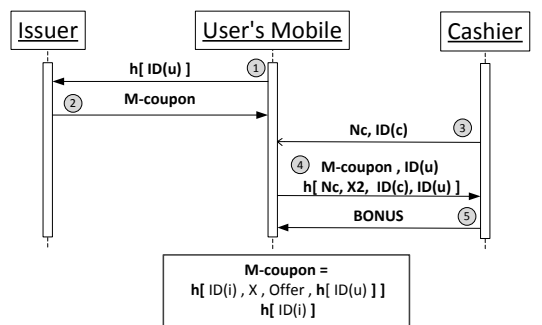

(b) the premium M-coupon protocol

Fig. 5. Suggested Solution

\section{Footfall/Premium Protocols notations:}

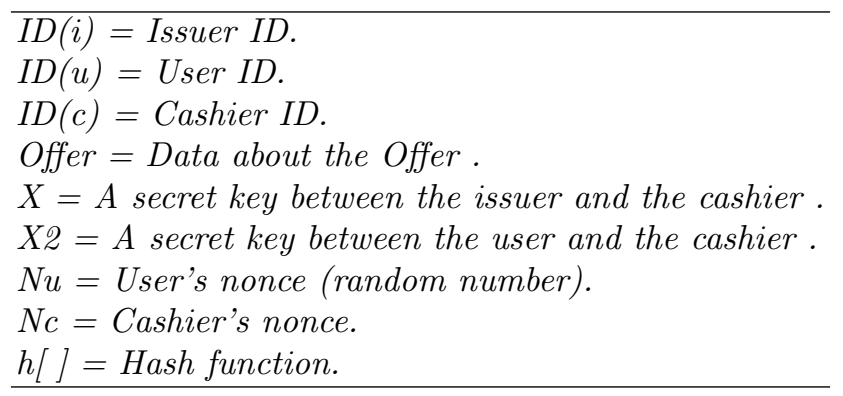

There are four messages in this protocol:

1. $U \rightarrow I: N u$

2. $I \rightarrow U: M$-coupon $=h[I D(i), X$, Offer,$N u], N u, h[I D(i)]$

3. $U \rightarrow C$ : M-coupon $=h[I D(i), X$, Offer,$N u], N u, h[I D(i)]$

4. $C \rightarrow U: B O N U S$

After the user brings his mobile close to the issuer, his mobile sends a random number $\mathrm{Nu}$ (message 1). Then, the Issuer sends the M-coupon to the user (message 2). The M-coupon contains a hash of the issuer identity, the secret key $\mathrm{X}$, the promised offer and user random number. In addition, a hash of the issuer identity, with the user's random number. Then, the user brings his mobile near the cashier and sends the M-coupon (message 3). From the hashes' table of issuers' identities, the cashier uses $\mathrm{h}[\mathrm{ID}(\mathrm{i})]$ to find the corresponding $\mathrm{ID}(\mathrm{i})$, secret $\mathrm{X}$ and the offer. The cashier can check the validity of the M-coupon. Through the nonce $\mathrm{Nu}$ the cashier can manage the M-coupon that every issued M-coupon has a unique random number. The cashier can, for example, stop using the Mcoupon after five uses. Finally, if all these conditions are satisfied, then bonus is given to the user (message 4 ). 
Confidentiality, Data Integrity and Forgery Protection requirements are ensured by use of the secret value $X$ and offer. Multiple Cash-in can be managed by including the nonce. However, a stronger Multiple Cash-in is provided in the premium M-coupon protocol.

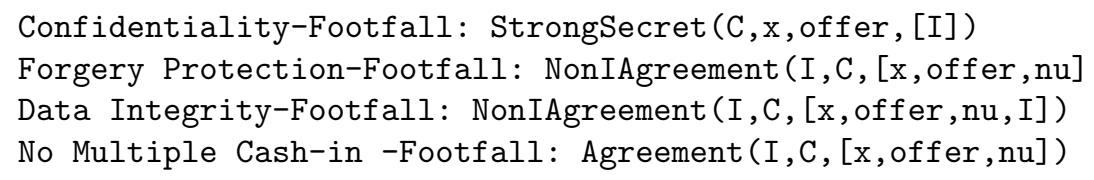

The premium M-coupon protocol The main enhancement in this protocol is attaching an authentic user identity to the coupon. I.e. addressing Not Transferable and User Authentication. Figure 5b illustrates the premium M-coupon protocol.

1. $U \rightarrow I: h[I D(u)]$

2. $I \rightarrow U: M$-coupon

3. $C \rightarrow U: N c, I D(c)$

4. $U \rightarrow C:$ M-coupon, $I D(u), h[N c, X 2, I D(c), I D(u)]$

5. $C \rightarrow U: B O N U S$

$M$-coupon $=h[I D(i), X$, Offer,$h[I D(u)]], h[I D(i)]$

The user's mobile sends a hash of his identity $\operatorname{ID}(\mathrm{u})$ to the issuer (message 1). Then, the Issuer sends the M-coupon to the user (message 2). The M-coupon contains a hash of: the issuer identity, the secret X, the offer, the hashed user's identity. In addition, a hash of the issuer identity. At cashing phase, the cashier sends his identity and a nonce Nc (message 3). At message 4, the user sends the M-coupon and the user identity, with a new hash contains: Nc, the secret value $\mathrm{X} 2$, the cashier identity and the user identity.

The cashier can send the bonus based on verifying the two hashes in message 4. From the hashes' table of issuers' identities, the cashier uses h[ID(i)] to find the corresponding $\operatorname{ID}(\mathrm{i})$, secret $\mathrm{X}$ and the offer, with user identity known from message 4 , the cashier can check the validity of the M-coupon. The second hash which authenticates the user, the cashier uses $\operatorname{ID}(\mathrm{u})$ to find the corresponding secret X2, and combines it with already known data (Nc, $\operatorname{ID}(\mathrm{c}), \operatorname{ID}(\mathrm{u}))$ to check the validity of the second hash. The cashier can link the M-coupon hash with the second one by checking that both of them include the same identity $\operatorname{ID}(u)$.

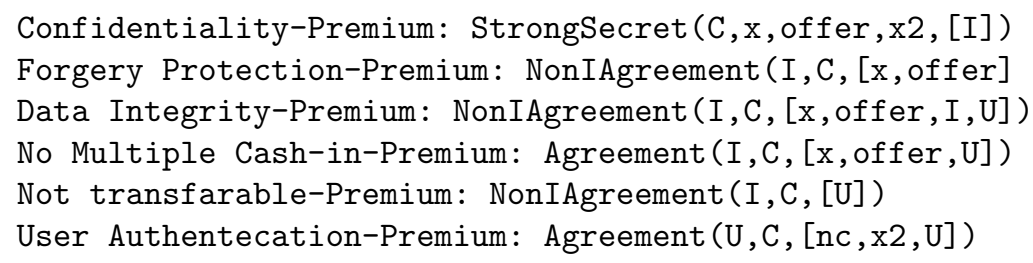

We formally verify the security of these solutions: the Casper/FDR2 analysis found no attacks. 


\section{Conclusion}

We used formal methods, Casper/FDR2, to examine a hash based M-coupon protocol and check whether it meets its requirements. The outcome of the analysis shows a simple logical attack in the hashes combination of the M-coupon, which damages many of protocol's requirements. Solutions were provided based on two assumptions: when the issuing phase is trusted where the intruder is more restricted; and where the intruder has the power to claim any identity. This paper can be considered as a case study of how a black-box analysis can provide powerful results about NFC protocols.

\section{References}

1. ISO/IEC: Information technology - telecommunications and information exchange between systems - near field communication - interface and protocol (NFCIP-1) (2004)

2. Finkenzeller, K.: RFID Handbuch:Fundamentals and Applications in Contactless Smart Cards, Radio Frequency Identification and Near-Field Communication. Third edn. John Wiley and Sons, Ltd. (2010)

3. Haselsteiner, E., Breitfuß, K.: Security in near field communication (NFC). In: Proceedings of Workshop on RFID and Lightweight Crypto (RFIDSec06). (2006)

4. Mulliner, C.: Vulnerability analysis and attacks on NFC-enabled mobile phones. In: ARES. (2009) 695-700

5. Juniper Research: Mobile coupons - ecosystem analysis and marketing channel strategy 2011-2016. Technical report, Juniper Research (2011)

6. Clark, S.: Survey: Discounts and coupons will drive adoption of mobile payments (2011) Avalible: http://www.nfcworld.com/2011/06/23/38289/ survey-discounts-and-coupons-will-drive-adoption-of-mobile-payments.

7. Smart Card Alliance: Proximity mobile payments business scenarios: Research report on stakeholder perspective. Technical report, Smart Card Alliance (2008)

8. Brown, C.: The future is NFC says coupons.com exec (2011) Available :http://www.nfcworld.com/2011/03/10/36399/ the-future-is-nfc-says-coupons-com-exec/.

9. Wolverton, T.: Disney battles coupon goof (2002) Available :http://news.cnet. com/2100-1017-964831.html.

10. Hsiang, H.C., Shih, W.K.: Secure mcoupons scheme using nfc. In: International Conference on Business and Information. (2011)

11. Lowe, G.: An attack on the Needham-Schroeder public-key authentication protocol. Inf. Process. Lett. 56(3) (1995) 131-133

12. Lowe, G.: Casper: A compiler for the analysis of security protocols. Journal of Computer Security 6(1-2) (1998) 53-84

13. Hoare, C.A.R.: Communicating Sequential Processes. Prentice-Hall (1985)

14. Ryan, P.Y.A., Schneider, S.A., Goldsmith, M., Lowe, G., Roscoe, A.W.: Modelling and analysis of security protocols. Addison-Wesley-Longman (2001)

15. Donovan, B., Norris, P., Lowe, G.: Analyzing a library of security protocols using Casper and FDR. In: Proceedings of the Workshop on Formal Methods and Security Protocols. (1999) Some of the Casper scripts are available here: http://web. comlab.ox.ac.uk/oucl/work/gavin.lowe/Security/Papers/prots.tar.gz.

16. Dolev, D., Yao, A.: On the security of public-key protocols. IEEE Transactions on Information Theory 2(29) (1983) 